\title{
QUASI-PERIODIC ACCELERATION OF ELECTRONS IN THE FLARE ON 2012 JULY 19
}

\author{
Jing Huang $^{1,2}$, Eduard P. Kontar ${ }^{3}$, Valery M. Nakariakov ${ }^{4}$, and Guannan GaO ${ }^{5}$ \\ ${ }^{1}$ Key Laboratory of Solar Activities, National Astronomical Observatories, Chinese Academy of Sciences, Beijing, 100012, China; huangj@bao.ac.cn \\ ${ }^{2}$ Sate Key Laboratory of Space Weather, Chinese Academy of Sciences, Beijing, 100190, China \\ ${ }^{3}$ School of Physics and Astronomy, University of Glasgow, Glasgow G12 8QQ, UK \\ ${ }^{4}$ Physics Department, University of Warwick, Coventry CV4 7AL, UK \\ ${ }^{5}$ Yunnan Observatory, Chinese Academy of Sciences, Kunming, Yunnan 650011, China \\ Received 2016 January 18; revised 2016 August 23; accepted 2016 August 23; published 2016 November 3
}

\begin{abstract}
Quasi-periodic pulsations (QPPs) of nonthermal emission in an M7.7 class flare on 2012 July 19 are investigated with spatially resolved observations at microwave and HXR bands and with spectral observations at decimetric, metric waves. Microwave emission at $17 \mathrm{GHz}$ of two footpoints, HXR emission at $20-50 \mathrm{keV}$ of the north footpoint and loop top, and type III bursts at $0.7-3 \mathrm{GHz}$ show prominent in-phase oscillations at $270 \mathrm{~s}$. The microwave emission of the loop leg has less pulsation but stronger emission. Through the estimation of plasma density around the loop top from EUV observations, we find that the local plasma frequency would be $1.5 \mathrm{GHz}$ or even higher. Thus, type III bursts at $700 \mathrm{MHz}$ originate above the loop top. Quasi-periodic acceleration or injection of energetic electrons is proposed to dominate these in-phase QPPs of nonthermal emission from footpoints, loop top, and above. In the overlying region, drifting pulsations (DPS) at $200-600 \mathrm{MHz}$ oscillate at a distinct period $(200 \mathrm{~s})$. Its global structure drifts toward lower frequency, which is closely related to upward plasmoids observed simultaneously from EUV emission. Hence, nonthermal emission from overlying plasmoids and underlying flaring loops show different oscillating periods. Two individual systems of quasi-periodic acceleration of electrons are proposed to coincide in the bi-direction outflows from the reconnection region.
\end{abstract}

Key words: Sun: oscillations - Sun: radio radiation - Sun: UV radiation

\section{INTRODUCTION}

In solar flares, quasi-periodic pulsations (QPPs) are usually observed markedly in nonthermal emission, such as radio, hard-X-ray (HXR), and gamma-ray wavelengths (Nakariakov \& Melnikov 2006, 2009; Nakariakov et al. 2010). Nonthermal emission is proposed to be produced by energetic particles when they are accelerated or transported into the solar atmosphere via bremsstrahlung or free-free emission, gyrosynchrotron emission, plasma emission, and so on (Aschwanden 2004). The sources distribute in a vast space in the solar atmosphere from chromosphere to corona, even to the interplanetary space. Based on the observational features of QPPs at multi-wavelength, the spatial and temporal distribution of these oscillations at different altitudes could be investigated. This would help us to distinguish the related oscillating mode and to obtain a new diagnosis on quasi-periodic acceleration or injection of particles in solar flares.

Solar radio bursts related to energetic electrons are observed in a wide frequency range from about tens of megahertz to tens of gigahertz. With the imaging observations from the Nobeyama radioheliograph (NoRH), the flaring loops have been resolved and studied (Asai et al. 2001; Melnikov et al. 2002, 2005; Grechnev et al. 2003; Nakariakov et al. 2003; Inglis et al. 2008; Kupriyanova et al. 2010, 2013; Kim et al. 2012). Asai et al. (2001) first presented the imaging features of QPPs from Nobeyama and Yohkoh observations. They estimated the Alfvén transit time along the flare loop and found that it was almost equal to the period of QPPs. Based on the shock acceleration model, they suggested that the variations of macroscopic magnetic structures, such as oscillations of coronal loops, could affect the efficiency of particle injection or acceleration. Inglis et al. (2008) studied a spatially resolved QPP event from microwave and HXR observations. They found that all parts of the loop in microwaves were seen to oscillate with the same period and almost in phase. One alternative interpretation is that the acceleration of nonthermal electrons is periodically modulated to generate periodic variation of nonthermal electron density, which modulates both microwave and HXR emission. Another option is the sausage mode of MHD oscillations in flare loops, which could modulate microwave emission by periodic variation of magnetic field in emitting plasma and modulate HXR emission by periodic change of the mirror ratio.

In some QPP events, more than one period is identified in the same burst process (Melnikov et al. 2005; Inglis \& Nakariakov 2009; Tan et al. 2010). Inglis \& Nakariakov (2009) reported a multi-periodic event of three distinct periods at 28 , 18 , and $12 \mathrm{~s}$. Considering the period ratios of this QPP event, they concluded that the cause of this multi-periodic event is likely to be a kink mode periodically triggering magnetic reconnection. Tan et al. (2010) reported another multi-periodic microwave QPPs at $2.6-3.8 \mathrm{GHz}$ with five distinct periods: $0.02-0.09,0.1-0.4,1.2-8,15-70$, and 220-270 s from the observation of Chinese Solar Broadband Radio Spectrometers at Huairou (SBRS/Huairou). They proposed that the first two groups of QPPs with short periods could be caused by resistive tearing-mode oscillations in current-carrying flare loops, while the other QPPs with long periods should be produced by MHD oscillations in different scales of magnetic configuration. These facts implied that the multi-scale of magnetic reconnection and energy release occur simultaneously in the flare region.

QPPs at microwave, HXR, and gamma-ray frequently oscillate in phase (Asai et al. 2001; Grechnev et al. 2003; Nakariakov et al. 2010). This suggests that both energetic electrons and ions are accelerated in the same process. Time delays between emission peaks have also been found from observations at different wave bands, which could be caused by trap or escape processes of energetic electrons at different 
energy bands (Aschwanden et al. 1997; Dolla et al. 2012; Tsap et al. 2013). Huang et al. (2014) found QPPs with varying periods in multi-wavelength observations in an $\mathrm{X}$ class flare. The oscillating period of microwave emission at low altitude is shorter than that of metric type III bursts at high altitude. It is proposed that kink oscillations of coronal loops at different altitudes with multiple scales are triggered simultaneously in the flare process.

From radio spectral observations, plenty of fine structures (FSs) were recorded with various configurations (Karlický et al. 2001; Wang et al. 2001; Huang et al. 2008; Huang \& Tan 2012). Coherent plasma emission is proposed to play a dominant role at decimeter and meter wavelengths, which may be associated with the flare primary energy-releasing process (Karlicky et al. 2001). Type III bursts are well-known at metric and decametric wavelengths. They often coincide with enhanced HXR emission, which is generally interpreted as the signature of energetic electron beams propagating along the magnetic field (Arzner \& Benz 2005). Hence, it could trace the path of beams from the near acceleration site toward the final destination as long as they are capable of producing radio emission (Benz 2004). Quasi-periodic pulsations with whole structure drifting toward high or low frequency are usually recorded in CME related events, which are interpreted as a signature of the dynamic phase of magnetic reconnection in a large-scale current sheet and the movement of plasmoids (Kliem et al. 2000; Karlický et al. 2004). In addition, the polarization of radio emission could provide us with more information on the orientation of magnetic field lines and the direction of waves. Foullon et al. (2010) found that the rightand left-handed circular polarized signals were periodically alternating at $1.36-1.48 \mathrm{GHz}$ from SBRS. They proposed that the source is located at the loop top and the changes of polarization could be due to a quasi-periodic transverse motion of the loop. Therefore, the analysis of spectral features of FSs could help us to diagnose the magnetic field, plasma, and energetic electrons in emitting sources to further understand the physical process of energy release and particle acceleration or transportation in flare regions.

The purpose of this paper is to study the QPPs of nonthermal emission in a limb flare-CME event on 2012 July 19, from spatially resolved microwave, HXR and EUV observations and dynamic spectral observations at decimetric and metric wavelengths. Based on the analysis of spatial and temporal distributions of QPPs in nonthermal emission, new evidence for quasi-periodic particle acceleration is obtained. Section 2 shows the observational features of QPPs at different wavebands. Section 3 includes the analysis and discussion. The summary and conclusions are presented in Section 4.

\section{OBSERVATIONS AND DATA REDUCTION}

The M7.7 flare on 2012 July 19 in AR 11520 (S13W88) started at 04:17 UT, peaked at 05:58 UT, and ended at 06:56 UT. NoRH (Nakajima et al. 1994) obtained microwave images at 17 and $35 \mathrm{GHz}$ and Nobeyama Radio Polarimeters (NoRP; Nakajima et al. 1985) recorded microwave total intensity at six fixed frequencies $(1.0,2.0,3.75,9.4,17$, and $35 \mathrm{GHz}$ ). SBRS recorded type III bursts at $2.6-3.8 \mathrm{GHz}$ with $8 \mathrm{~ms}$ temporal resolution and $10 \mathrm{MHz}$ frequency resolution. Decimetric and metric digital solar radio spectrometers of Yunnan Astronomical Observatories (YNRS; Gao et al. 2014) observed type III bursts and spikes at $625-1500 \mathrm{MHz}$ and DPS

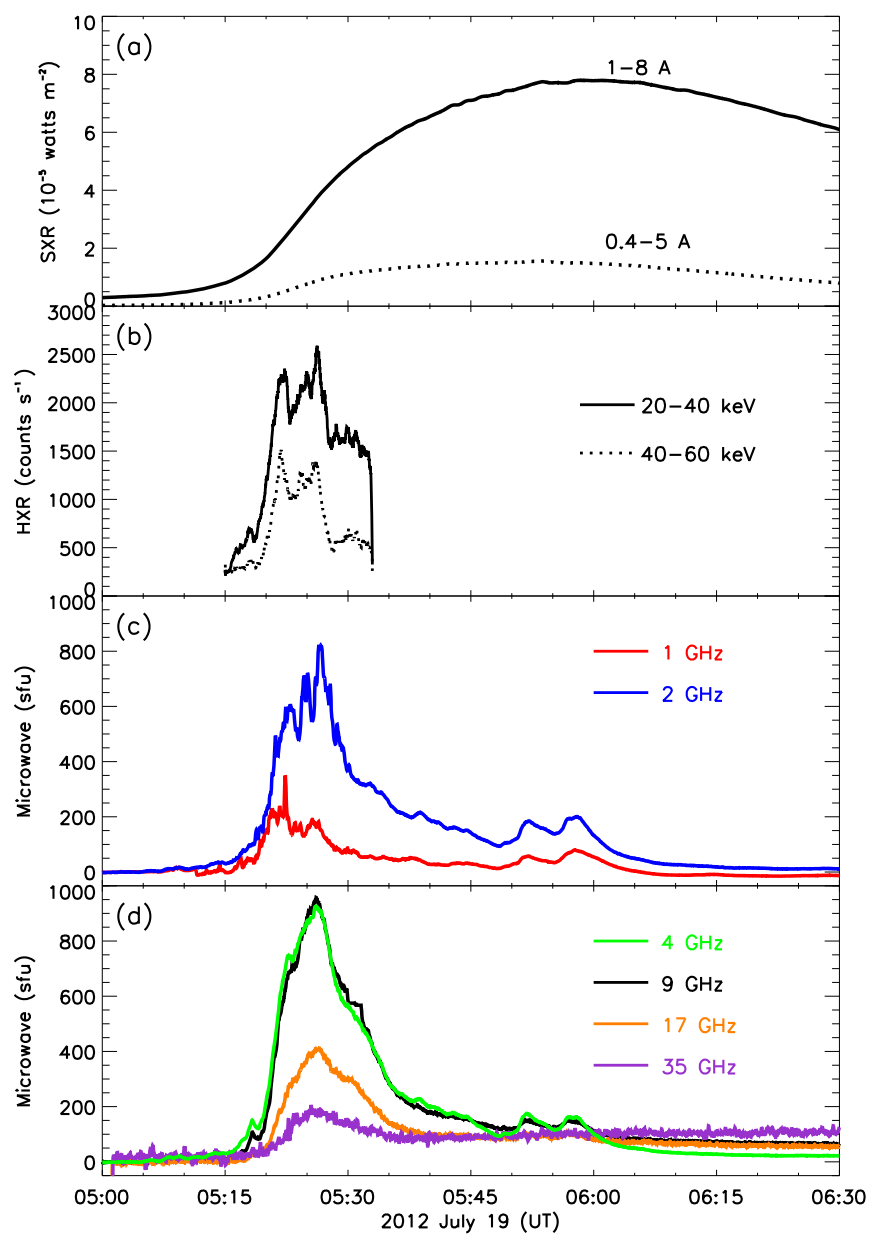

Figure 1. Total intensity of SXR emission at $1-8$ and $0.4-5 \AA$ (a), HXR emission at 20-40 and 40-60 keV (b), and microwave emission at 1, 2, 4, 9, 17 , and $35 \mathrm{GHz}((\mathrm{c})$ and (d)).

at $70-700 \mathrm{MHz}$ with $0.2 \mathrm{MHz}$ spectral resolution and $80 \mathrm{~ms}$ temporal resolution. Atmospheric Imaging Assembly (AIA) instrument of SDO took full-disk images of the Sun in seven EUV channels and three UV-visible channels with high spatial and temporal resolutions ( 0 ". 6 and $12 \mathrm{~s})$. The total intensity of SXR, HXR, and microwave emission are plotted in Figure 1. Both HXR and microwave emission obviously increase during the rise phase of the SXR flare. Furthermore, HXR emission at $20-60 \mathrm{keV}$ and microwave emission at 1,2 , and $4 \mathrm{GHz}$ oscillate quasi-periodically with several peaks. The wavelet analysis shows that the spectral power enhances at about 100 and $270 \mathrm{~s}$ (Figure 2). For the microwave emission at high frequency $(9,17$, and $35 \mathrm{GHz})$, the fluxes smoothly increase and decrease without obvious oscillations.

\subsection{Microwave Observation}

Figure 3 presents the microwave images at 17 and $34 \mathrm{GHz}$, recorded by NoRH. The evolution of the microwave flaring loop could be identified as three phases. Before the obvious increase of microwave emission at $17 \mathrm{GHz}$ (at about 05:17 UT), the microwave flare loop was formed and gradually grew up, while the emission was very weak. During this phase, microwave emission was enhanced around the loop top. In the second phase, from 05:17 to 05:39 UT, there was a dark region around the loop top and the emission enhanced at loop legs. 
(a) $2 \mathrm{GHz}$

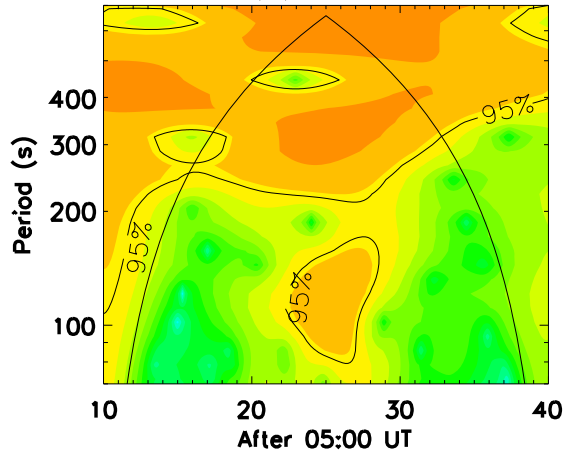

(b) $4 \mathrm{GHz}$

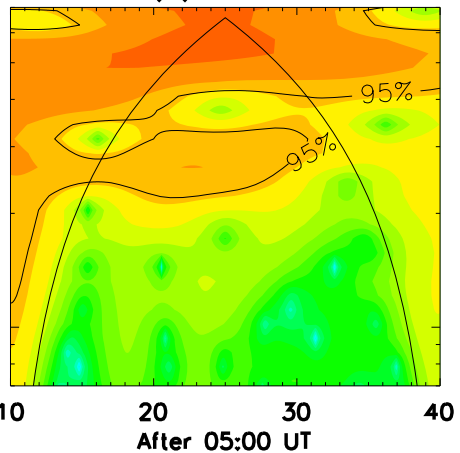

(c) $40-60 \mathrm{keV}$

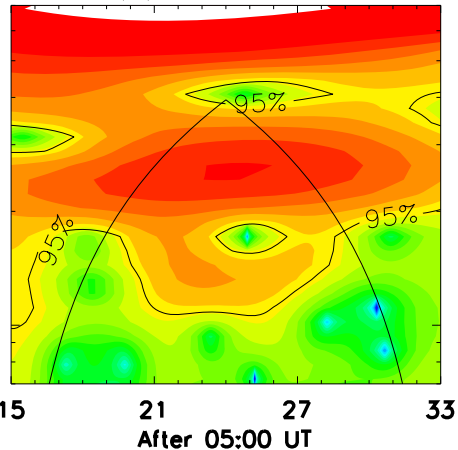

Figure 2. Wavelet analysis of total intensity of microwave emission at 2 and $4 \mathrm{GHz}$ and $\mathrm{HXR}$ emission at $40-60 \mathrm{keV}$.
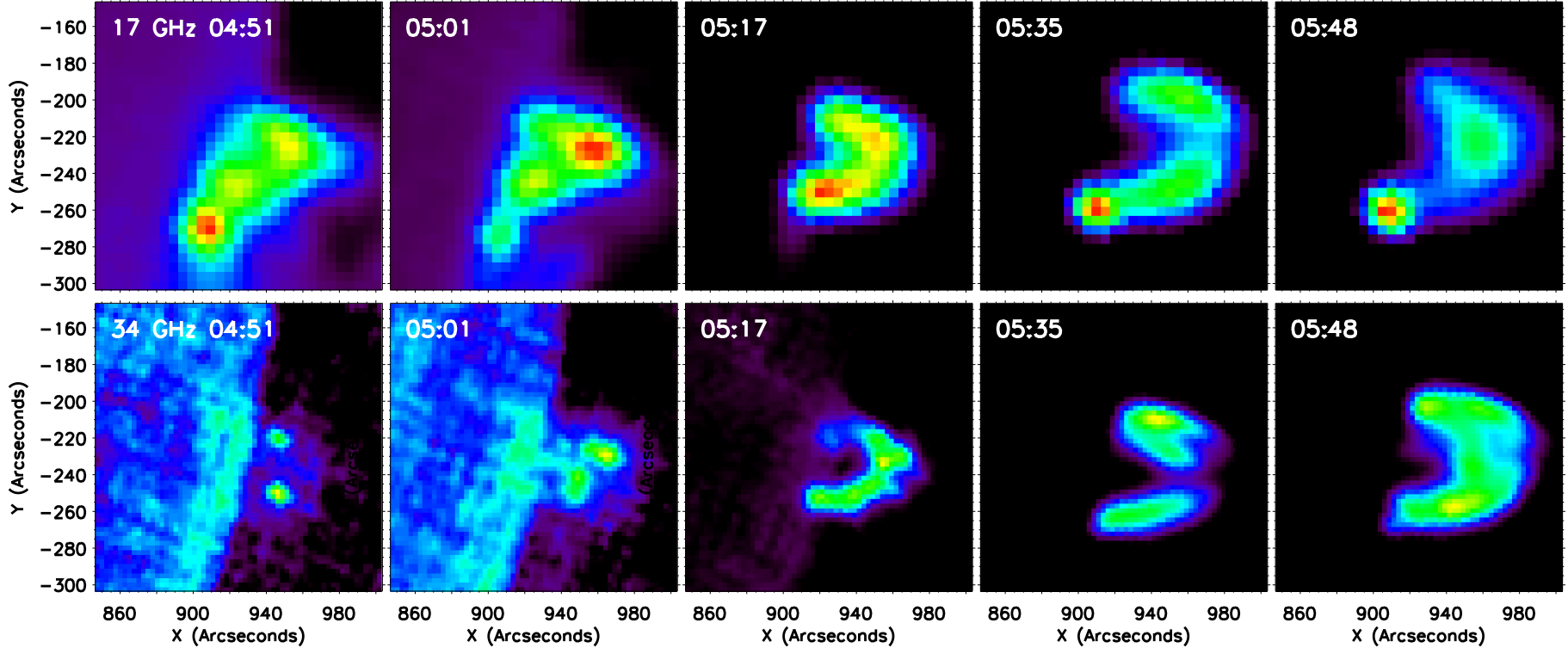

Figure 3. Microwave images at 17 and $34 \mathrm{GHz}$ during the flare process.

After 05:39 UT, the whole loop was visible and the loop top became the brightest region. A bright point source near the south foot point (SFP) at $17 \mathrm{GHz}$ existed in the whole event. The oscillations of microwave total intensity appear in the second phase.

In order to find oscillations from resolved images and study their distribution along the flare loop, four regions were selected in each loop leg (Figure 4). To eliminate the influence of noise on the data, the background is deleted by subtracting the mean flux integrated over the quiet region. The pixel with a value less than $1 \%$ of the maximum in each image is eliminated. The mean flux of these considered pixels in each selected region is plotted in Figure 5. It is found that the emission from SO, S1, and N0 shows obvious oscillations and the wavelet spectrum power is enhanced at about $100 \mathrm{~s}$ and $270 \mathrm{~s}$ (Figure 6). The other selected regions have smooth light curves and the emission from loop legs have the strongest emission.

\subsection{HXR Observation}

Both loop-top (LT) and foot-point sources could be distinguished in HXR observations. The contours of HXR

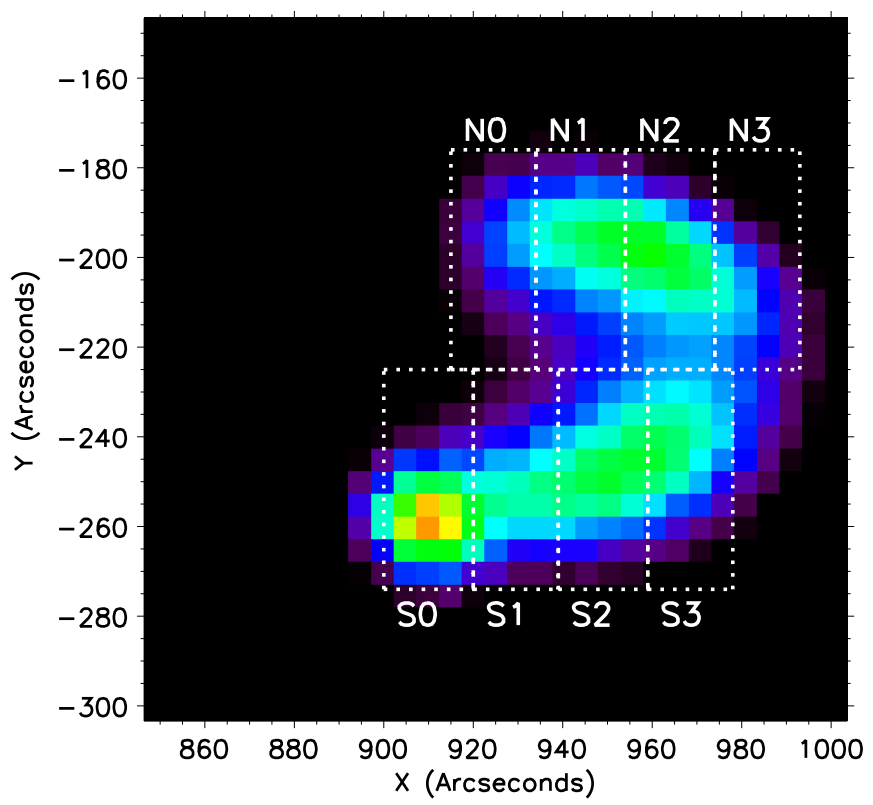

Figure 4. Selected regions of the microwave loop at $17 \mathrm{GHz}$ at 05:35 UT. 

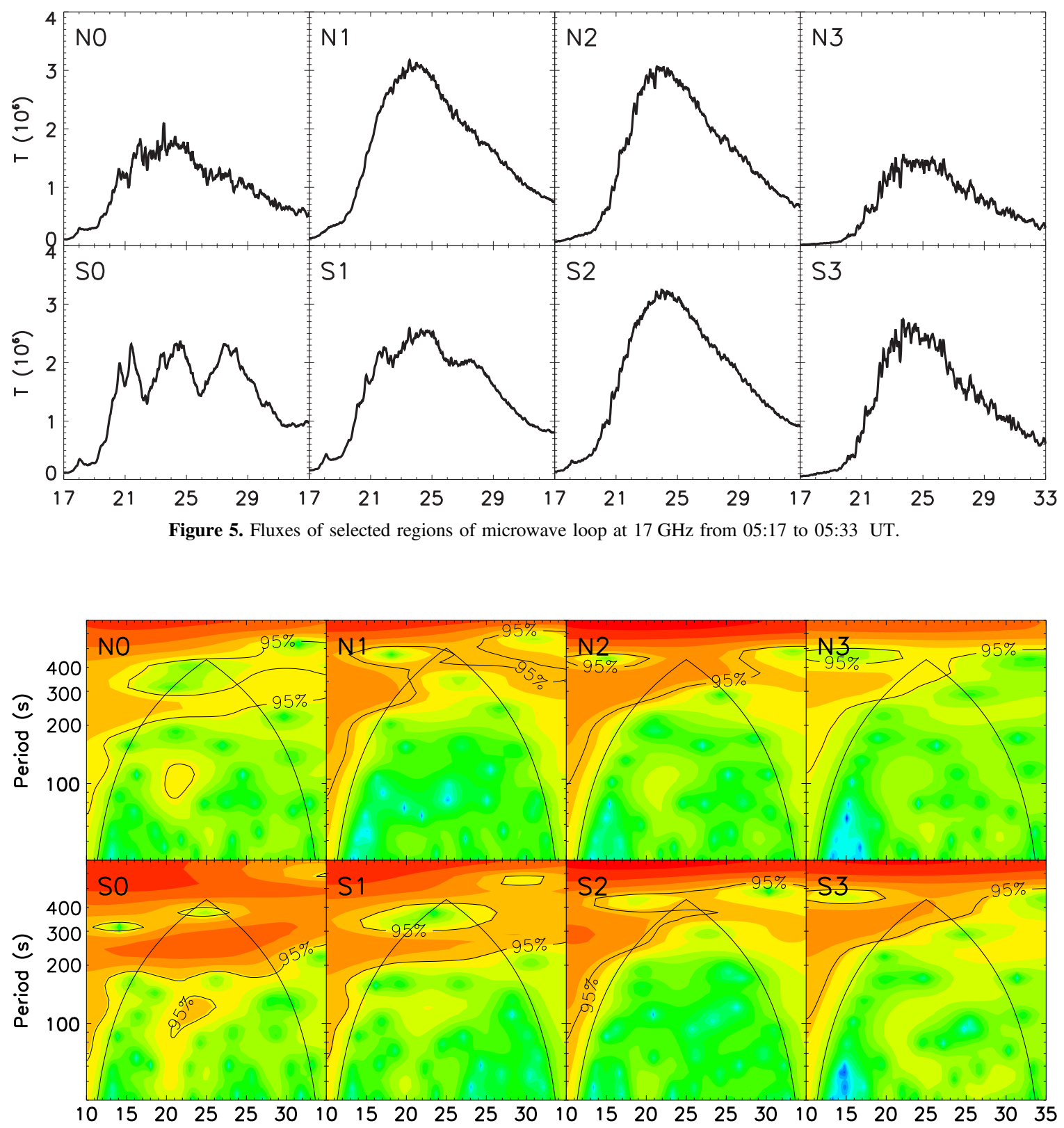

Figure 6. Wavelet analysis of selected regions of the microwave loop at $17 \mathrm{GHz}$ from 05:10 to 05:35 UT.

sources at four energy bands are superposed on the microwave image at $17 \mathrm{GHz}$ (Figure 7). The LT source appears at 10-20, 20-30, and 30-40 keV and FP sources are detected at 20-30, $30-40$, and $40-50 \mathrm{keV}$. Because the south foot point (SFP) is very weak and could not be distinguished clearly at times, we focus on the study of LT and north FP (NFP). The fluxes of LT and NFP integrated in $20 \mathrm{~s}$ are plotted in Figure 8. The emission of LT at 10-20 keV was smoothly increased from 05:18 to 05:33 UT, which could be the signal of heating of local plasma. At $20-30 \mathrm{keV}$, the flux of LT presents as three peaks superposing on a smoothly increasing background. The slow changing of the background is due to thermal emission while the impulsive peaks represent nonthermal emission emitted directly by energetic electrons. At the other energy bands, the light curves of both LT and NFP have three distinct peaks with an oscillating period of $270 \mathrm{~s}$ as NFP at $20-30 \mathrm{keV}$, and NFP has stronger emission.

\subsection{Decimetric and Metric Observations}

The spectra at three frequency bands recorded by SBRS and YNRS are plotted in Figure 9. From about 05:20 to 05:30 UT, type III bursts at $2.6-3.0 \mathrm{GHz}$ are observed by SBRS. Each individual burst drifts from low to high frequency with a drifting rate of about $4 \mathrm{GHz} \mathrm{s}^{-1}$. At $0.7-1.5 \mathrm{GHz}$, YNRS observed type III bursts from 05:10 to 05:33 UT. It is not capable of calculating the frequency rate for an individual burst with $80 \mathrm{~ms}$ resolution at this waveband. At $200-700 \mathrm{MHz}$, pulsations with whole structure drifting toward low frequency are recorded from 05:13 to 05:30 UT. The frequency drifting rate is about $0.56 \mathrm{MHz} \mathrm{s}{ }^{-1}$. The wavelet analysis of the light curve at $540 \mathrm{MHz}$ of DPS and the one at $1060 \mathrm{MHz}$ of type III bursts shows that DPS has quasi-periodic oscillations at about 70 and $200 \mathrm{~s}$ and type III bursts have oscillating periods of 100 and $270 \mathrm{~s}$ (Figure 10). 


\subsection{Summary of Observations}

Table 1 summarizes the observational features of QPPs in nonthermal emission at multi-wavelength. Radio emissions at microwave, decimetric, and metric waves and HXR emission at 20-50 keV show obvious oscillations. Using microwave and

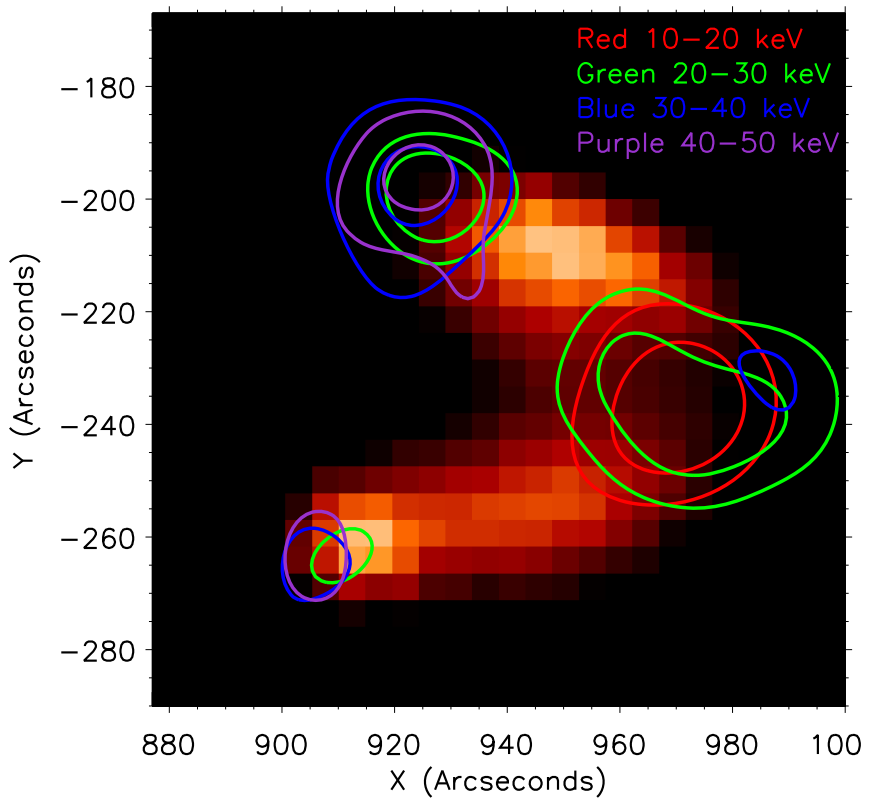

Figure 7. Contours of HXR sources at 10-20 (red), 20-30 (green), 30-40 (blue), and 40-50 (purple) keV, overlapping above the microwave loop at $17 \mathrm{GHz}$.
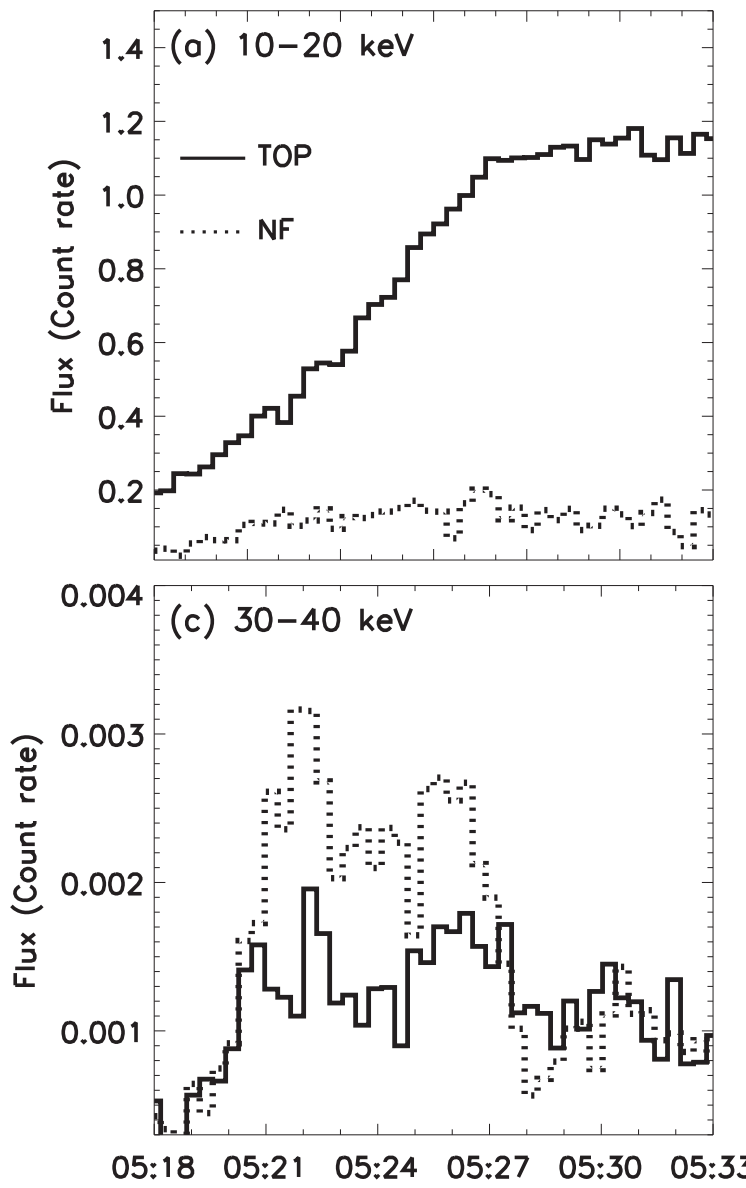

HXR images of this limbic flare, the spatial distribution of QPPs could be obtained. The oscillations at 100 and $270 \mathrm{~s}$ dominate at FPs (SFP and NFP of microwave emission and NFP of HXR emission) and LT (HXR emission). Part of the south loop leg (S1) shows weak oscillations. Type III bursts at $0.7-1.5 \mathrm{GHz}$ and $2.6-3.0 \mathrm{GHz}$ are seen to oscillate with a similar period as microwave and HXR emissions. DPS at 200-700 MHz has an entirely different oscillating period of 70 and $200 \mathrm{~s}$. The periods at 200 and $270 \mathrm{~s}$ could be the fundamental components of oscillations and 70 and $100 \mathrm{~s}$ are the harmonic ones, respectively. The left panel of Figure 11 shows the light curves of type III bursts at $1060 \mathrm{MHz}$, HXR LT, and NFP at $30-40 \mathrm{keV}$, type III bursts at $2.6 \mathrm{GHz}$ and microwave SFP at $17 \mathrm{GHz}$. Here we only want to compare the evolution of emission at different wavebands and do not plot their units. It shows clearly the in-phase evolution of emission among different wavebands. The right panel shows the crosscorrelation coefficients between HXR LT (solid lines) and NFP (dotted lines) fluxes and the light curves at $1060 \mathrm{MHz}$, and 2.6 and $17 \mathrm{GHz}$. They have good correlation with each other and the oscillations at $270 \mathrm{~s}$ could be displayed clearly.

\section{ANALYSIS}

\subsection{The Features of Nonthermal Emission}

\subsubsection{FP Sources}

HXR FP sources are generally the most prominent nonthermal emissions during solar flares and are frequently observed as pairs or ribbon-like structures (Dennis et al. 2011; Holman et al. 2011;
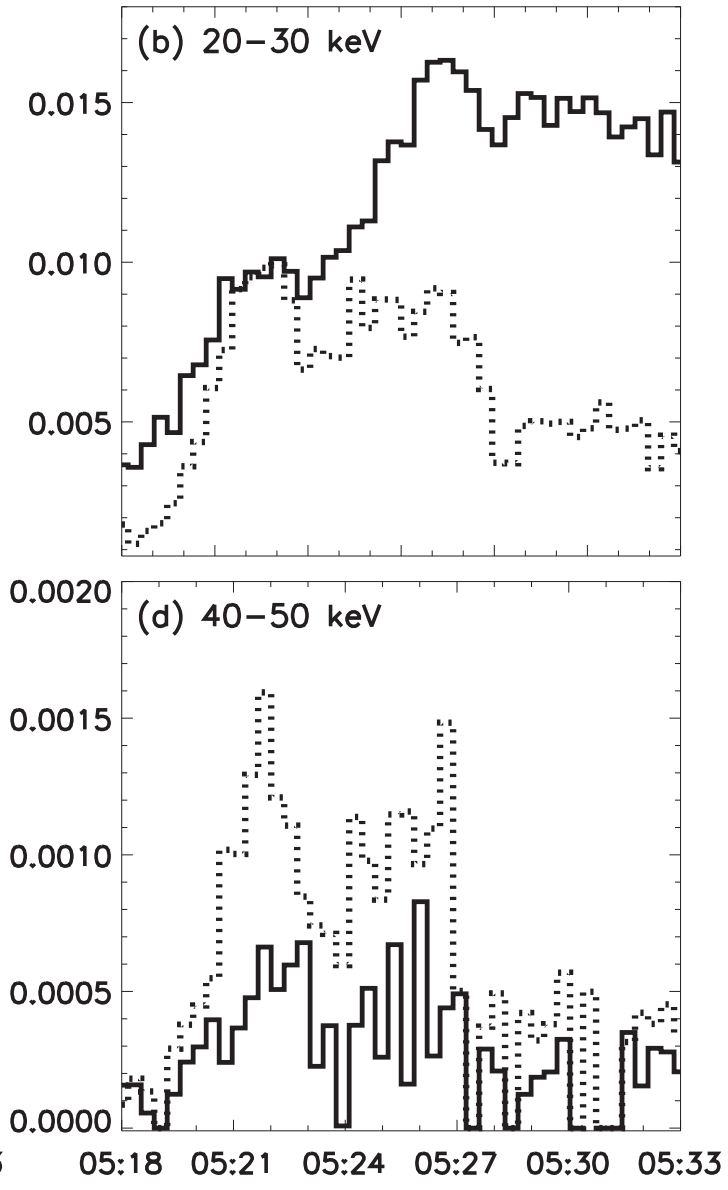

Figure 8. Fluxes of HXR LT and NFP at four energy bands. 


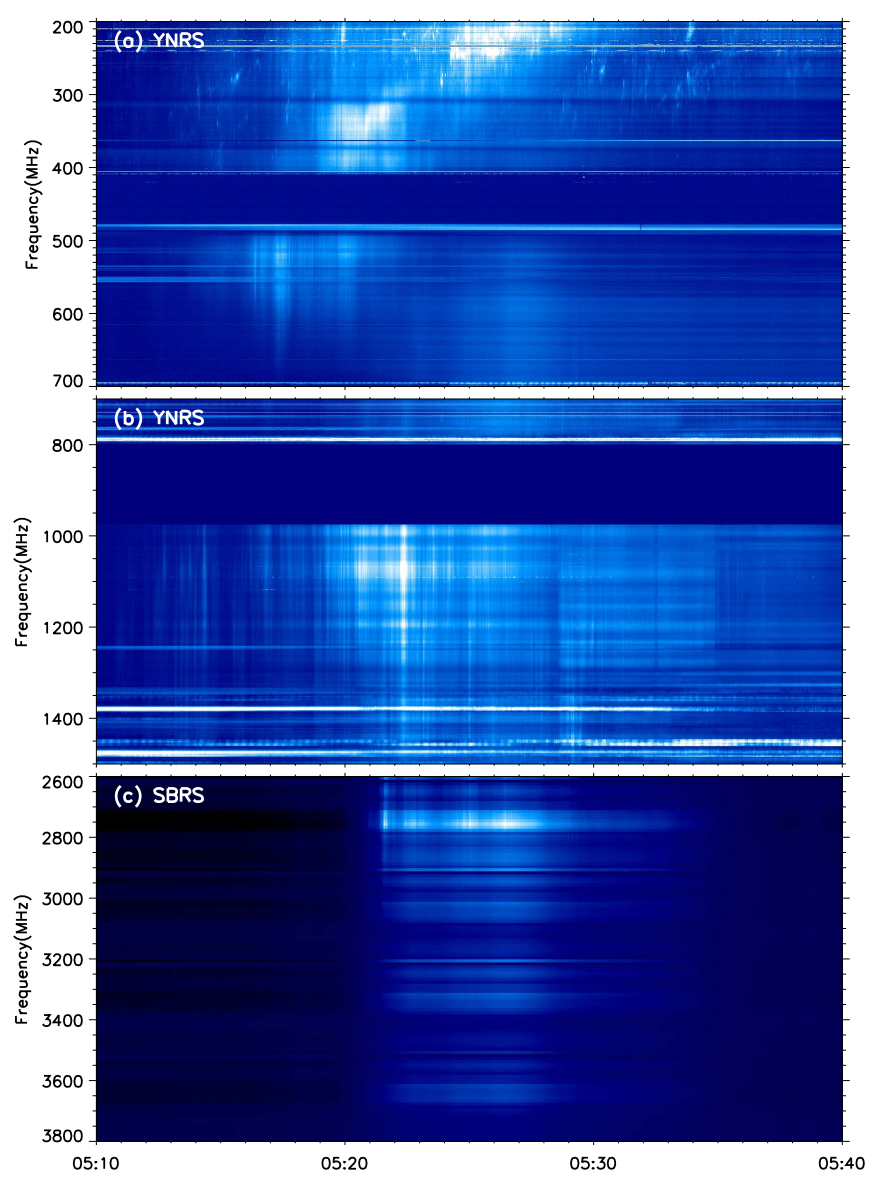

Figure 9. Decimetric and metric spectra observed by SBRS and YNRS.

Kontar et al. 2011). They are emitted via a thick-target bremsstrahlung mechanism when energetic electrons are collisionally decelerated by the plasma around FPs. Microwave emission at $17 \mathrm{GHz}$ would be produced by energetic electrons spreading all over the loop through the gyrosynchrotron mechanism. In an asymmetric magnetic loop, the observations always present unequal brightness. This phenomenon could be caused by different distributions of energetic electrons in the frame of the trap-plus-precipitation model for energetic electrons in solar flares (Bastian et al. 1998; Aschwanden 2004; Lee 2004).

In this event, HXR SFP almost overlaps microwave SFP, while HXR NFP deviates slightly from microwave NFP. Both HXR NFP and microwave SFP show prominent quasi-periodic evolution, and microwave NFP shows similar oscillations but less modulation depth. The different location and evolution of HXR and microwave NFP would indicate that they are emitted by different populations of energetic electron, which could also be interpreted in the frame of the trap-plus-precipitation model of energetic electrons. HXR NFP and microwave SFP could be emitted by precipitating electrons, which are located below magnetic mirror point. Because precipitating electrons include directly precipitating ones that are not trapped in the loop after injection and second precipitating ones that escape from trap, it is essential to distinguish which component plays the leading role in oscillatory emission. This is discussed in Section 3.2. Microwave NFP could be located near or above the magnetic mirror point and produced by both free-traveling and trapped electrons. The trapped electrons are accumulated gradually in the flaring loop and generate smooth emission, which reduce the oscillating amplitude of precipitating ones.

\subsubsection{Loop Legs}

It is known that the distribution of microwave radiation in the flaring loop is closely related to the configuration of the magnetic field, the distribution of energetic electrons, and the condition of local thermal plasma. Based on EUV observation of flare loops, Sun et al. (2014) calculated the distribution of emission measure (EM) and temperature $(T)$ along the flare loop. The values of EM and $T$ have no obvious differences between two legs, which suggest a similar condition of thermal plasma inside two loop legs. All of the selected regions, except for $\mathrm{S} 1$, in loop legs have smooth emission during the flare and the two loop legs have similar intensities. This futher suggests that there is a smooth increase of trapped electrons that dominate the emission in loop legs. Region S1, with weaker oscillations, could be in the same situation as that of NFP (N0).

\subsubsection{LT Source}

From 05:17 to 05:39 UT, the microwave LT was very weak. Morgachev et al. (2014) have performed a recovery of these flare loop parameters using NoRH and RHESSI data. The reconstruction of the loop top would be successful only for the anisotropic (quasi-longitudinal) electron pitch-angle distribution, which may suggest a longitudinal injection of accelerated electrons near the loop top. From the results of Sun et al. (2014), EM around LT at 05:24:12 UT is about $1.3 \times 10^{30} \mathrm{~cm}^{-5}$. By estimation of the size of LT from the EUV image, we estimate the density of thermal plasma as being about $3 \times 10^{10} \mathrm{~cm}^{-3}$. Then the local plasma frequency is about 1.5 GHz. Battaglia \& Kontar (2013) have performed a combined analysis of RHESSI and AIA data to infer the electron distribution function over the broad energy range from $0.1 \mathrm{keV}$ up to a few tens of $\mathrm{keV}$. From a case study, they found that the results deduced from AIA observations could be lower than that from the RHESSI fit. Based on this result, the local plasma frequency would be higher than $1.5 \mathrm{GHz}$. The emitting frequency of type III bursts is approximately equal to basic or second harmonic local plasma frequency, so type III bursts at $700 \mathrm{MHz}$ originate above LT. The emission of each individual burst, drifting from $700 \mathrm{MHz}$ to $3 \mathrm{GHz}$, implies that energetic electron beams depart from the acceleration site and move downward into the flaring loop to generate type III bursts on their way when the radiation condition could be satisfied. This is the signature of the downward injection of accelerated electrons.

\subsubsection{Plasmoid Ejection}

From EUV images, the eruption of plasmoids could be clearly observed (Liu et al. 2013). The upward ejections have a median initial velocity of $320 \mathrm{~km} \mathrm{~s}^{-1}$ and a maximum of $1050 \mathrm{~km} \mathrm{~s}^{-1}$. DPS is first recorded at 05:13 UT and occurs simultaneously with an ejection with the velocity at $1050 \mathrm{~km} \mathrm{~s}^{-1}$. Its whole structure drifts toward low frequency, which indicates an upward movement of the source with the local plasma density decreasing. If we set the length scale of local plasma to $2 \times 10^{10} \mathrm{~cm}^{-1}$, the velocity of the DPS source could be $900 \mathrm{~km} \mathrm{~s}^{-1}$. This could be comparable with the plasmid at 05:11 UT, observed from EUV images. Hence, DPS 
(a) $540 \mathrm{MHz}$ of DPS

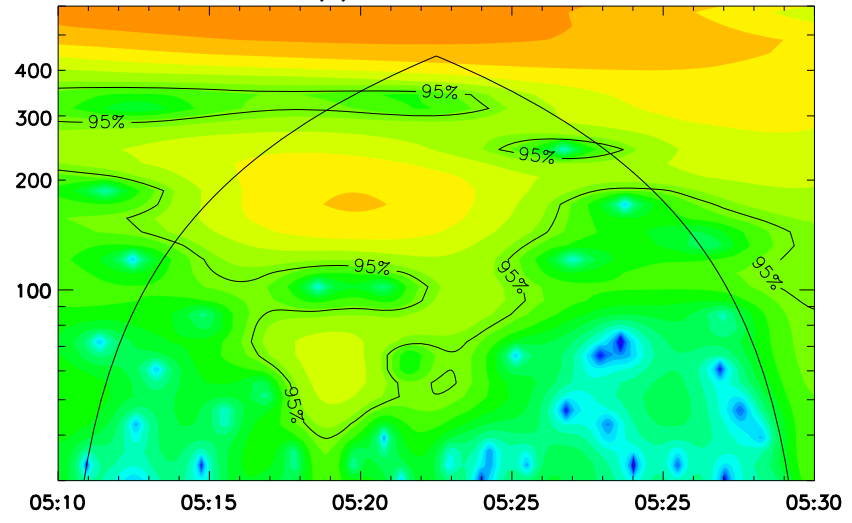

(b) $1060 \mathrm{MHz}$ of type III

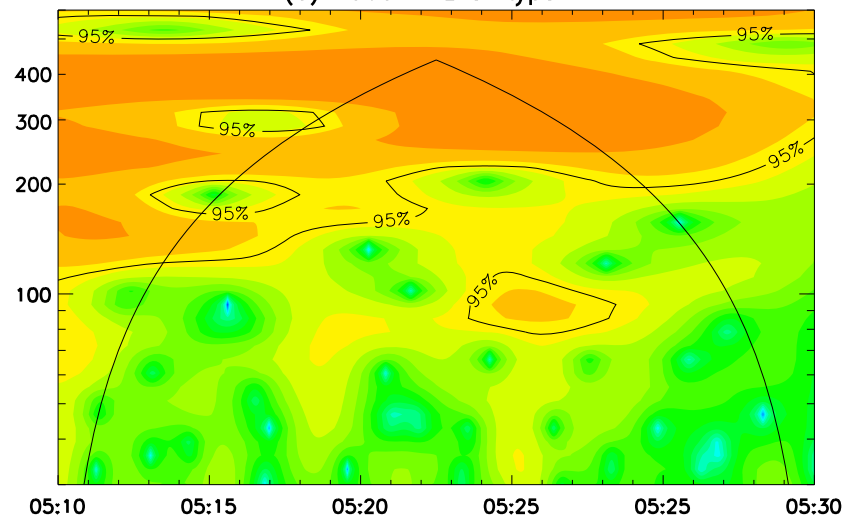

Figure 10. Wavelet analysis of DPS at $540 \mathrm{MHz}$ and type III bursts at $1060 \mathrm{MHz}$.

Table 1

Brief Summary of Multi-wavelength QPPs

\begin{tabular}{lcll}
\hline \hline Wavelength & Period (s) & \multicolumn{1}{c}{ Source } & Instrument \\
\hline $17 \mathrm{GHz}$ & 100,270 & SFP, NFP and S1 & NoRH \\
$20-50 \mathrm{keV}$ & 270 & NFP, LT & RHESSI \\
$2.6-3.0 \mathrm{GHz}$ & 100,270 & Type III bursts & SBRS \\
$0.7-1.5 \mathrm{GHZ}$ & 100,270 & Type III bursts & YNRS \\
$200-700 \mathrm{MHz}$ & 70,200 & DPS & YNRS \\
\hline
\end{tabular}

is closely related with plasmoids. The individual pulsation in DPS is also emitted by energetic electron beams, which may indicate the process of injection or acceleration in plasmoids.

\subsection{Oscillating Origin for Nonthermal Emission}

Nonthermal emission in solar flares is the immediate production of energetic electrons by various mechanisms when they travel in the solar atmosphere. Liu (2013) has proposed an initial, slow acceleration and subsequent, impulsive acceleration in this flare process. QPPs of nonthermal emission appear in the phase of impulsive acceleration, when both HXR and microwave emission obviously enhanced. During this phase, nonthermal emission from FP, LT, and the above region shows in-phase oscillations at $270 \mathrm{~s}$, and that in plasmoids at high altitude oscillates at $200 \mathrm{~s}$. The MHD oscillation of flare loops would be one possible option to understand the above features. It could result in the oscillatory evolution of the magnetic field, thermal plasma, or energetic electrons to modulate the emission in flare loops. In this case, SXR and EUV emission would also show oscillations, which are not be detected from observations. Furthermore, only FPs and region S1 in the flare loop show oscillations in microwave emission. The emission of type III bursts in the injection region above LT has similar oscillations as that of LT and FPs. Thus, MHD oscillation of flare loops is insufficient to interpret above features.

Another alternative possibility is quasi-periodic injection or acceleration of energetic electrons. In such a situation, the oscillatory number of energetic electrons traveling in the solar atmosphere is the principle factor in quasi-periodic emission. In this event, the oscillatory feature of type III bursts and HXR LT exactly suggests a quasi-periodic process of injection or acceleration of electrons at $270 \mathrm{~s}$. The in-phase oscillation between type III bursts and the emission from FPs implies that they are related to the same population of energetic electrons. Thus, we could distinguish oscillatory first precipitating electrons from smooth secondary precipitating ones. The first precipitating electrons, namely, partial of injected electrons, travel freely toward FPs and produce oscillatory microwave and HXR emission there. Hence, the quasi-periodic injection or acceleration of downward energetic electrons are responsible to QPPs of nonthermal emission in underlying flaring loops.

At high altitude, the nonthermal emission in plasmoids shows another oscillatory period, which may suggest another process of quasi-periodic injection or an acceleration process. Thus, two distinct oscillations are detected in nonthermal emission, originating from different locations in the same flaring process. There should be two independent systems to modulate the injection or the acceleration process at different altitudes, which occur outside of the common reconnection region. Liu et al. (2013) found bi-direction outflows originating between an erupting flux rope and underlying flare loops. They suggested that the primary loci of particle acceleration and plasma heating are in the reconnection outflow regions, rather than the reconnection site itself. Our results are consistent with the above result and obtain further evidence of different oscillatory periods of these two acceleration processes. The oscillatory energetic electrons produced by quasi-periodic acceleration at $270 \mathrm{~s}$ in downward outflows is responsible to nonthermal emission in the underlying flare loop, and the quasi-periodic acceleration of electrons at $200 \mathrm{~s}$ in upward plasmoids generates DPS in the overlying region.

\section{SUMMARY AND DISCUSSION}

The limb flare on 2012 July 19 provide us with an opportunity to study the spatial distribution of QPPs of nonthermal emission. From imaging and spectral observations at multi-wavelengths, including HXR, microwave, decimetric, and metric waves, QPPs are observed in a wide region from FPs, LT to plasmoids, and two distinct periods were detected in this flare region. The observational features and analysis are summarized as follows.

The flaring loop structure could be determined by a microwave observation, which evolves in three phases. QPPs appear at the second phase with intensive emission from loop legs and weak emission around LT. We analyze the fluxes of selected regions in flaring loop and find that the emissions of FPs and region S1 have oscillations at $270 \mathrm{~s}$. The loop legs have smoother light curves but stronger emission. This difference could be caused by different populations of energetic electrons, which could be understood in the frame of the trap-plus-precipitating model of energetic electron in solar flares. The intensive-smooth emission 


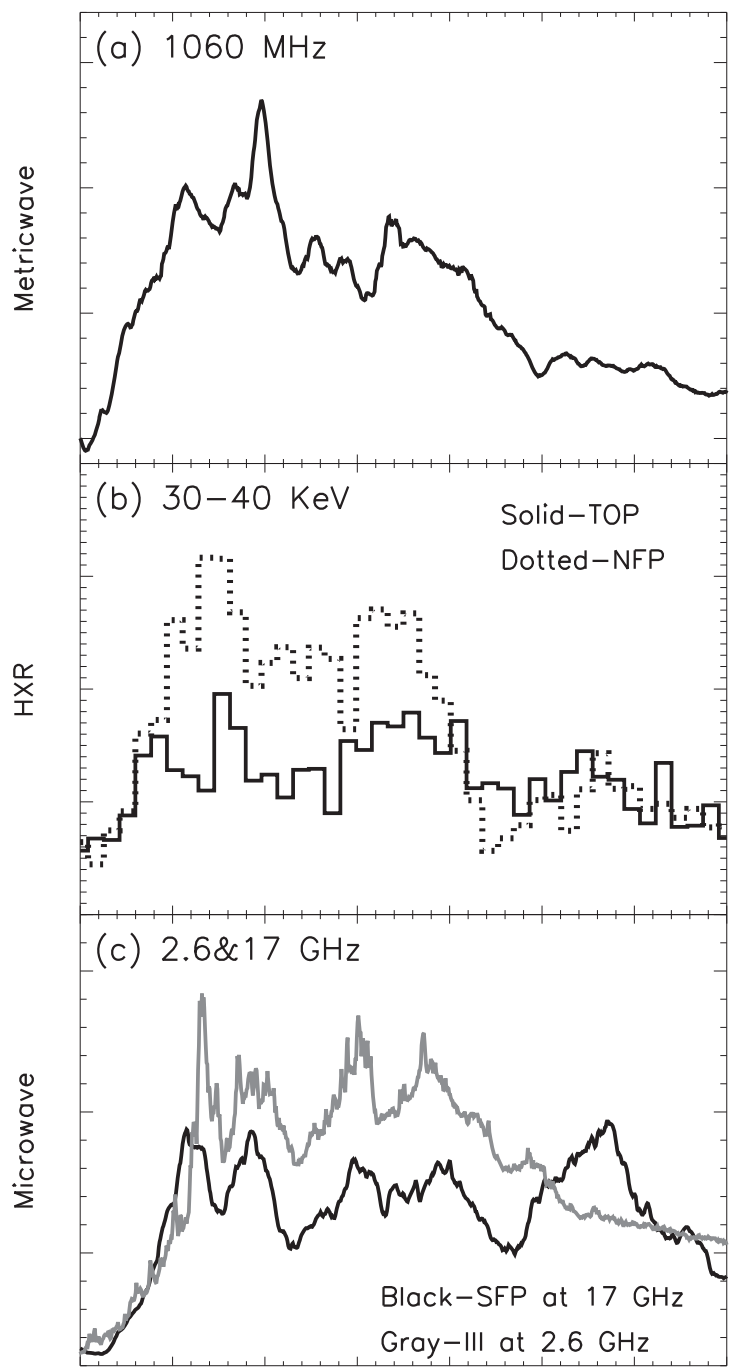

05:19 05:21 05:23 05:25 05:27 05:29 05:30 05:33 - $\quad$ 0 400

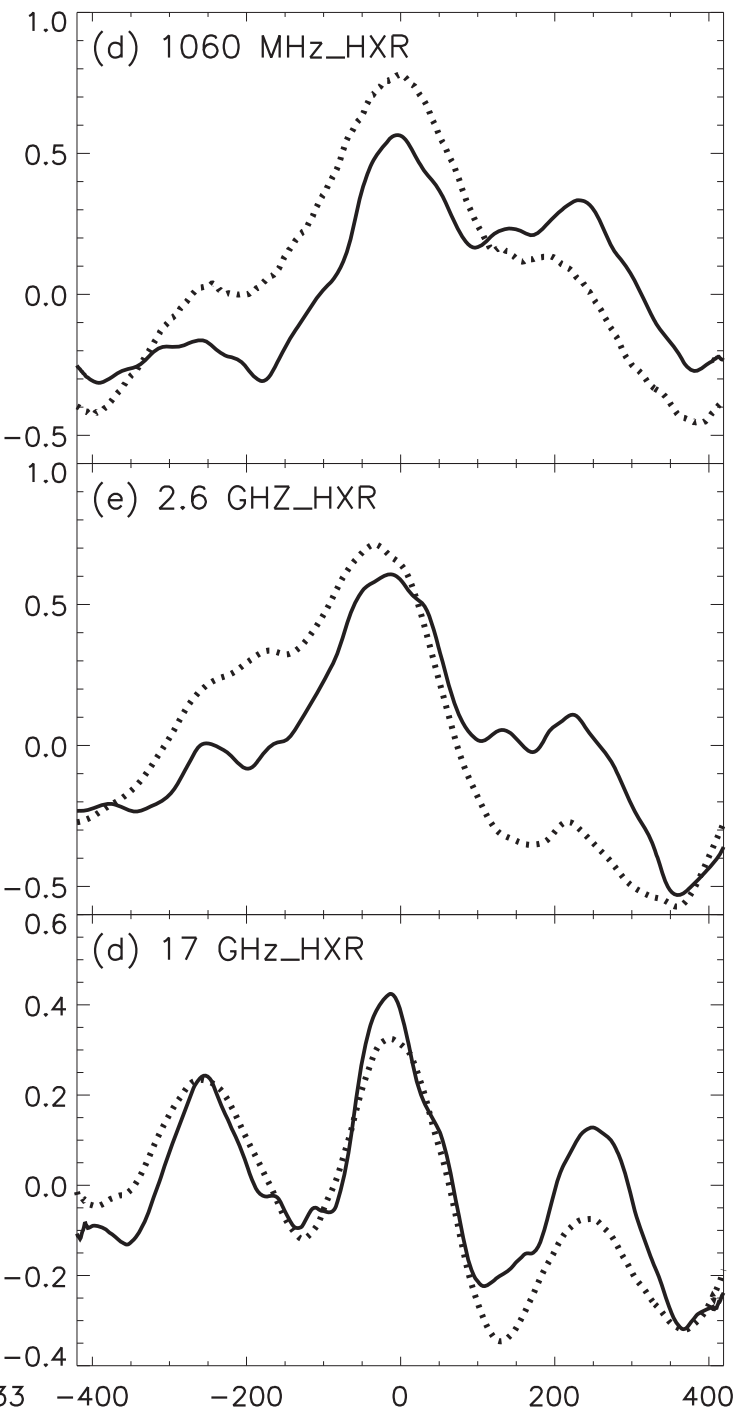

Figure 11. Left panel: light curves of emission at $1060 \mathrm{MHz}, 2.6$ and $17 \mathrm{GHz}$, and HXR LT and NFP at 30-40 keV. Right panel: the results of the cross-correlation between emission at $1060 \mathrm{MHz}, 2.6$ and $17 \mathrm{GHz}$, and HXR LT (solid lines) and NFP (dotted lines).

of loop legs could be dominated by a greater number of accumulated trapped electrons, while the oscillating emission from FPs and S1 is modulated by the oscillating change of first precipitating electrons.

The HXR emissions observed by RHESSI have two FPs and an LT. The emission of LT at $10-20 \mathrm{keV}$ is increased continuously, which is thermal emission, reflecting the heating of local plasma by injected energetic electrons. The one at $20-30 \mathrm{keV}$, increasing with oscillatory peaks at $270 \mathrm{~s}$, is a mixture of thermal and nonthermal emission. The nonthermal emission at $30-50 \mathrm{keV}$ of LT and NFP shows obvious oscillation at a period of $270 \mathrm{~s}$. Both would be dominated by an oscillatory number of energetic electrons, which implies a quasi-periodic process of injection or acceleration of electrons.

From spectral observations, quasi-periodic type III bursts with periods of $270 \mathrm{~s}$ cover a wide range at $0.7-3 \mathrm{GHz}$. The individual type III burst drifts toward high frequency, which indicates a downward movement of energetic electron beams. The local plasma frequency near LT is estimated as about $1.5 \mathrm{GHz}$ or even higher frequency. This indicates that the emission of type III bursts at lower frequency originated from above LT and energetic electrons, oscillating at $270 \mathrm{~s}$, transport downward from the acceleration site to LT, loop legs, and FPs. The well-related fluxes between type III bursts and HXR LT and NFP suggests a common origin for them. DPS at 200-600 MHz, occurring simultaneously with an upward plasmoid at high altitude, have an oscillating period of $200 \mathrm{~s}$. The similarity of the velocity of DPS source and plasmoids indicates that they are closely related to each other. It means that the energetic electrons in plasmoids oscillate at a distinct period.

The above observations and implications provide us with an understanding of electron acceleration and transportation in this flare. The energetic electrons accelerated in this flare produce quasi-periodic nonthermal emission at two distinct periods in underlying flare loops and overlying plasmoids, respectively. MHD oscillations of flaring loops is excluded by the observed features of smooth fluxes at EUV and SXR bands. Quasiperiodic acceleration or injection of energetic electrons could be a reasonable option. Two individual processes of acceleration are proposed to take place at different locations out of the reconnection site. The electrons quasi-periodically accelerated at $270 \mathrm{~s}$ in downward outflows generate in-phase oscillations of HXR LT and type III bursts. Furthermore, the ones precipitating 
directly to FPs, partial of injected ones, emit quasi-periodic microwave and HXR emission there. In upward plasmoids, electrons are quasi-periodically accelerated at $200 \mathrm{~s}$, which subsequently produce oscillatory DPS. The outflows from the reconnection site disturb the local plasma and generate MHD oscillations, which modulates the acceleration process. The different condition in local plasma would result in distinct periods.

Actually, it is difficult to distinguish between the processes of acceleration and injection. It is essential to diagnose the oscillatory parameters in the acceleration or injection region to find the main modulating factor. Due to the lack of imaging observations of type III bursts and DPS, the locations of these sources could not be obtained. It is difficult to estimate the parameters and find the origin of oscillations. Ming'antu Spectral Radio Heliograph (MUSER), formerly called the Chinese Solar Radio Heliograph (CSRH; Yan et al. 2009), would provide high spatial-temporal-frequency observations of solar activities at $400 \mathrm{MHz}-15 \mathrm{GHz}$. It may improve this study to obtain more information on quasi-periodic injection or acceleration of electrons.

The authors would like to thank Marian Karlicky for helpful comments and all of the teams for providing observation data. This work was supported by NSFC Grant 11573039, 11273030, 11221063, 11103044, 11373039, the National Major Scientific Equipment R\&D Project ZDYZ2009-3, the Specialized Research Fund for State Key Laboratories No. 15010009 (2015-25F-04), and the Grant P209/12/0103 (GA CR). This work was also supported by the Marie Curie PIRSES-GA295272-RADIOSUN project.

\section{REFERENCES}

Arzner, K., \& Benz, A. O. 2005, SoPh, 231, 117

Asai, A., Shimojo, M., Isobe, H., et al. 2001, ApJL, 562, L103

Aschwanden, M. J. 2004, in Physics of the Solar Corona. An Introduction (Chichester, UK: Praxis)

Aschwanden, M. J., Bynum, R. M., Kosugi, T., Hudson, H. S., \& Schwartz, R. A. 1997, ApJ, 487, 936

Bastian, T. S., Benz, A. O., \& Gary, D. E. 1998, ARA\&A, 36, 131

Battaglia, M., \& Kontar, E. P. 2013, ApJ, 779, 107
Benz, A. O. 2004, in Solar and Space Weather RadioPhysics-Current Status and Future Development, ed. D. E. Gary \& C. U. Keller (Dordrecht: Kluwer), 206

Dennis, B. R., Emslie, A. G., \& Hudson, H. S. 2011, SSRv, 159, 3

Dolla, L., Marqué, C., Seaton, D. B., et al. 2012, ApJL, 749, L16

Foullon, C., Fletcher, L., Hannah, I. G., et al. 2010, ApJ, 719, 151

Gao, G., Wang, M., Dong, L., Wu, N., \& Lin, J. 2014, NewA, 30, 68

Grechnev, V. V., White, S. M., \& Kundu, M. R. 2003, ApJ, 588, 1163

Holman, G. D., Aschwanden, M. J., Aurass, H., et al. 2011, SSRv, 159, 107

Huang, J., \& Tan, B. 2012, ApJ, 745, 186

Huang, J., Tan, B., Zhang, Y., Karlický, M., \& Mészárosová, H. 2014, ApJ, 791,44

Huang, J., Yan, Y. H., \& Liu, Y. Y. 2008, SoPh, 253, 143

Inglis, A. R., \& Nakariakov, V. M. 2009, A\&A, 493, 259

Inglis, A. R., Nakariakov, V. M., \& Melnikov, V. F. 2008, A\&A, 487, 1147

Karlický, M., Bárta, M., Jiřička, K., et al. 2001, A\&A, 375, 638

Karlický, M., Fárník, F., \& Krucker, S. 2004, A\&A, 419, 365

Kim, S., Nakariakov, V. M., \& Shibasaki, K. 2012, ApJL, 756, L36

Kliem, B., Karlický, M., \& Benz, A. O. 2000, A\&A, 360, 715

Kontar, E. P., Brown, J. C., Emslie, A. G., et al. 2011, SSRv, 159, 301

Kupriyanova, E. G., Melnikov, V. F., Nakariakov, V. M., \& Shibasaki, K. 2010, SoPh, 267, 329

Kupriyanova, E. G., Melnikov, V. F., \& Shibasaki, K. 2013, SoPh, 284, 559

Lee, J. 2004, in Solar and Space Weather RadioPhysics-Current Status and Future Development, ed. D. E. Gary \& C. U. Keller (Dordrecht: Kluwer), 182

Liu, R. 2013, MNRAS, 434, 1309

Liu, W., Chen, Q., \& Petrosian, V. 2013, ApJ, 767, 168

Melnikov, V. F., Reznikova, V. E., Yokoyama, T., et al. 2002, in Solar variability: From Core to Outer Frontiers. The 10th European Solar Physics Meeting, ed. A. Wilson (Noordwijk: ESA Publications Division), 339

Melnikov, V. F., Reznikova, V. E., Shibasaki, K., \& Nakariakov, V. M. 2005, A\&A, 439, 727

Morgachev, A., Melnikov, V., \& Kuznetsov, S. 2014, XL COSPAR Scientific Assembly, 40

Nakajima, H., Nishio, M., Enome, S., et al. 1994, IEEEP, 82, 705

Nakajima, H., Sekiguchi, H., Sawa, M., Kai, K., \& Kawashima, S. 1985, PASJ, 37,163

Nakariakov, V. M., Foullon, C., Myagkova, I. N., \& Inglis, A. R. 2010, ApJL, 708, L47

Nakariakov, V. M., \& Melnikov, V. F. 2006, A\&A, 446, 1151

Nakariakov, V. M., \& Melnikov, V. F. 2009, SSRv, 149, 119

Nakariakov, V. M., Melnikov, V. F., \& Reznikova, V. E. 2003, A\&A, 412, L7

Sun, J. Q., Cheng, X., \& Ding, M. D. 2014, ApJ, 786, 73

Tan, B., Zhang, Y., Tan, C., \& Liu, Y. 2010, ApJ, 723, 25

Tsap, Y., Kopylova, Y., Goldvarg, T., \& Stepanov, A. 2013, PASJ, 65, S6

Wang, S., Yan, Y., \& Fu, Q. 2001, A\&A, 370, L13

Yan, Y., Zhang, J., Wang, W., et al. 2009, EM\&P, 104, 97 